\title{
Unusual bilateral muscular variation in the medial forearm: separate humeral and ulnar bellies of flexor carpi ulnaris and anomalous muscle addition
}

\author{
Amanda M. Pugh, Brittni A. Silvestri, Matthew A. Ward, William M. Whited, Anthony A. Capehart \\ Department of Biology, East Carolina University, Greenville, NC, USA
}

\begin{abstract}
We report herein an unusual bilateral variation in musculature of the medial forearm discovered during routine anatomical dissection. On the left side there was complete separation of humeral and ulnar bellies of the flexor carpi ulnaris, while on the right there was an anomalous muscle addition from the medial epicondyle extending to the hamate. In the left forearm, tendons of both humeral and ulnar bellies remained separate until insertion on the pisiform and an extratendinous slip passed laterally from the humeral head to insert on the hamate. On the right, the flexor carpi ulnaris only had minor connection to the medial epicondyle and was essentially ulnar in origin. The anomalous muscle originated entirely from the medial epicondyle and was innervated predominantly by the median nerve. Before insertion on the hamate, an extratendinous slip passed medially to insert on the tendon of flexor carpi ulnaris. Additional variations associated with the flexor carpi ulnaris are discussed and importance of these muscular variants evaluated.
\end{abstract}

Key words: flexor carpi ulnaris; anatomical variation; humeral and ulnar heads

Anatomy 2010; 4: 67-71, ( ) 2010 TSACA

\section{Introduction}

Awareness of potential muscular variations is essential for anatomists, surgeons, and clinicians in numerous areas of medical concentration. In the upper extremity the flexor carpi ulnaris muscle originates from humeral and ulnar heads in the usual instance, with the humeral origin arising from the medial epicondyle and a more extensive origin of the ulnar head from the medial olecranon and posterior border of the ulna. The heads merge with the tendon inserting on the pisiform and through ligamentous attachments to the hamate and fifth metacarpal. ${ }^{1}$ While interesting unilateral variations in the flexor carpi ulnaris muscle have been recently reported, ${ }^{2,3}$ we describe here an unusual case in which there is bilateral separation of humeral and ulnar muscle bellies of the flexor carpi ulnaris in the left forearm accompanied by the presence of an abnormal flexor carpi ulnaris origin and an adjacent anomalous or accessory muscle on the right.

\section{Case Report}

Bilateral variation in the medial forearm musculature of a 76-year-old male was discovered during routine class dissection and preparation of specimens for nursing and allied health anatomy and physiology laboratories. As shown in Figures 1A and $\mathbf{C}$, the smaller belly of flexor carpi ulnaris arose on the left side from the medial epi- 
condyle along with other superficial muscles of the anterior compartment and was designated the humeral head. The larger belly arose largely from the olecranon and posterior border of the ulna, retaining only a thin aponeurotic connection to the medial epicondyle separate from the common flexor tendon and was thus labeled as the ulnar head. Both bellies received separate innervation from the ulnar nerve; the humeral head receiving a branch shortly after passage of the ulnar nerve around the medial epicondyle and a branch slightly distal to the ulnar head. Upon reflection of the ulnar attachment to the medial epicondyle, the ulnar nerve was observed to pass between the two bellies (Figure 1D). Tendons of both bellies began at approximately one third of their length from the origin, the smaller humeral head becoming completely tendinous at that point. The ulnar belly displayed a flattened tendon on its deep surface and otherwise remained muscular until approximately $1 \mathrm{~cm}$ proximal to its insertion (Figures 1A and B). Insertion of both tendons was on the pisiform, with the humeral tendon ventral to that of the ulnar belly and both remaining separate until their attachment. A small tendinous slip also extended lateralward from the humeral belly to insert on the hamate (Figure 1B), underneath which the ulnar artery and superficial branch of ulnar nerve passed into the hand.

As seen in Figures $2 \mathbf{A}$ and $\mathbf{E}$, the flexor carpi ulnaris in the right forearm had minor connection to the medial epicondyle separate from the common flexor tendon and muscle origin from the olecranon and posterior ulna was noted. Aside from the limited tendinous connection to the medial epicondyle the humeral head was lacking. The ulnar head became tendinous on its deep surface and remained muscular on its ventral aspect until shortly before insertion on the pisiform. The smaller head of the adjacent anomalous muscle addition arose entirely from the medial epicondyle in common with the other superficial musculature, became tendinous in its proximal portion and resembled strongly the humeral belly of flexor carpi ulnaris in the left forearm. Reflection of the minor origin of flexor carpi ulnaris to the medial epicondyle showed passage of the ulnar nerve between the ulnar belly and adjacent anomalous variant (Figure 2F). Innervation to the flexor carpi ulnaris was from a branch of the ulnar nerve entering the muscle in its proximal portion. Interestingly, the anomalous humeral muscle received several small branches of the median nerve on its lateral aspect and only a single small branch of the ulnar nerve was noted to enter the muscle at its origin from the medial epicondyle. In the right forearm the tendon of this muscle variant passed directly to the hamate and sent a small tendinous slip medialward to join the tendon of the ulnar belly of flexor carpi ulnaris on its underside just proximal to its insertion on the pisiform (Figures 2B and C). The superficial branch of the ulnar nerve entered the hand between the insertion of flexor carpi ulnaris and the anomalous variant. A small muscular slip originated from the base of the hypothenar eminence at the pisiform and passed laterally, deep to the superficial branch of the ulnar nerve to insert on the hamate along with the tendon of the humeral variant (Figures $\mathbf{2 B}$ and $\mathbf{D}$ ). As an additional note, palmaris longus was absent from the anterior compartment of the left forearm, although present on the right (Figures 1A and 2A).

\section{Discussion}

Reports of anatomical variation of the flexor carpi ulnaris or anomalous muscles associated with the medial forearm have been relatively few. ${ }^{4-8}$ Recently, however, an additional tendon of flexor carpi ulnaris was observed unilaterally, passing on its deep surface from the medial epicondyle to the pisiform. ${ }^{2}$ Moreover, documentation of separate humeral and ulnar muscle bellies and tendons of flexor carpi ulnaris that fused shortly before insertion on the pisiform in the left upper limb was the first reported digastric variant of flexor carpi ulnaris. ${ }^{3}$ It was suggested by these authors that this latter variation might be attributed to failure of muscle primordia to fuse during embryonic development, a theory supported by normal occurrence of separated primary nerve branches that distribute to humeral and ulnar segments of the flexor carpi ulnaris. ${ }^{9,10}$

In the present case we report bilateral variation of medial forearm musculature. In the left forearm the separation of humeral and ulnar bellies of flexor carpi 
Figure 1. Photographs of the anterior compartment of the left forearm illustrating separate humeral and ulnar bellies of the flexor carpi ulnaris. A: Overview of forearm anatomy showing origin of humeral and ulnar bellies and insertion on the pisiform (large arrowhead: humeral tendon; small arrowhead: ulnar tendon). A small extratendinous slip from the humeral tendon extends toward the hamate (long arrow). B: Closer view of humeral (large arrowhead) and ulnar (small arrowhead) insertion shown in A. The extratendinous slip from the humeral tendon arches over the superficial branch of the ulnar nerve and ulnar artery. C: Closer view of origin of humeral and ulnar bellies shown in A. Ulnar nerve is shown passing behind the medial epicondyle (me). D: Branch of the ulnar nerve is shown entering the ulnar portion as it passes between the two bellies (short arrow). H: humeral belly; U: ulnar belly; me: medial epicondyle; UN: ulnar nerve; UA: ulnar artery.

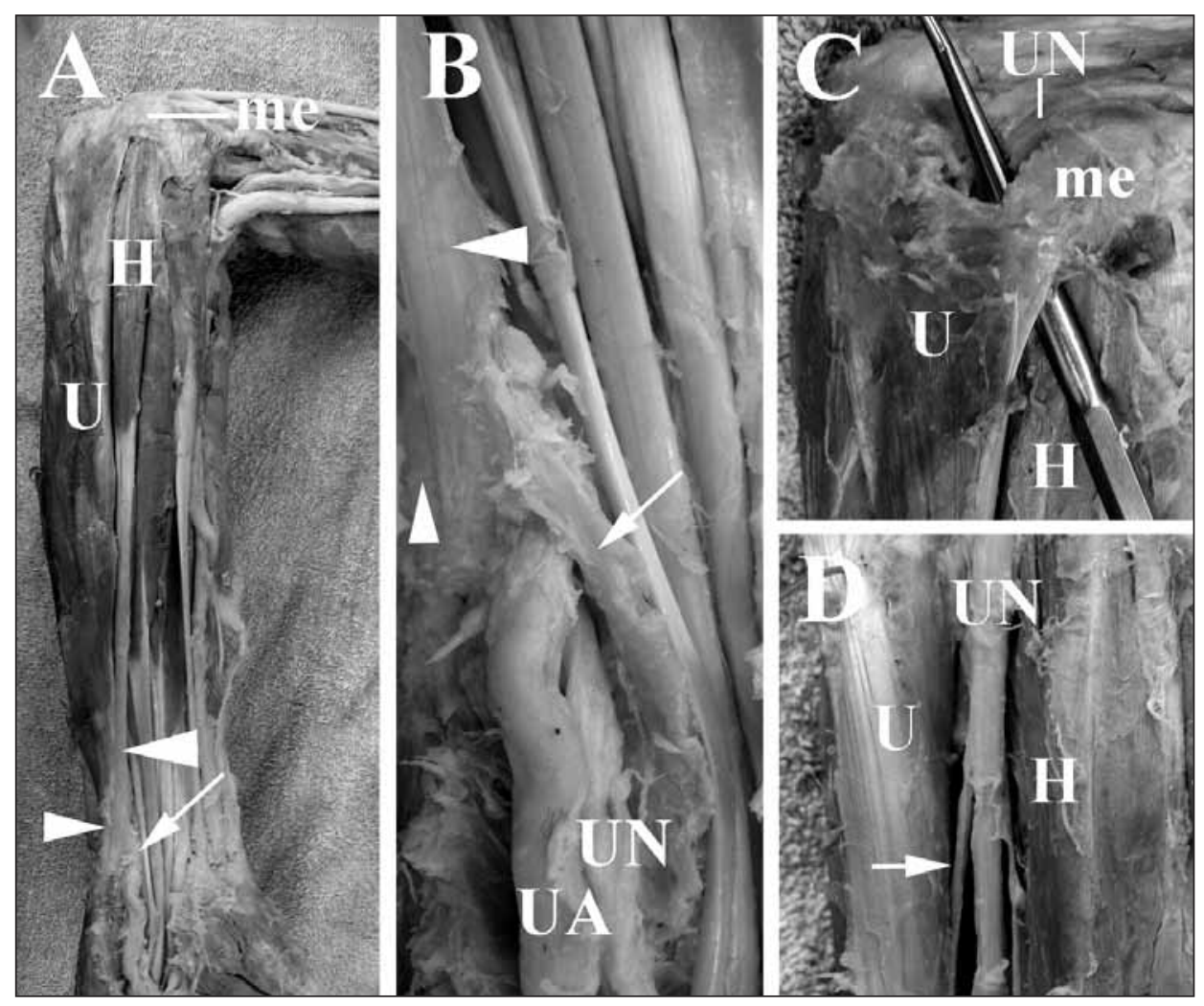

Figure 2. Photographs of the anterior compartment of the right forearm demonstrating the humeral variant and flexor carpi ulnaris muscles. A: Overview of forearm anatomy showing origin of humeral variant and ulnar belly of flexor carpi ulnaris and insertion of the humeral variant tendon (large arrowhead) on the hamate and ulnar tendon (small arrowhead) on the pisiform. A small slip from the humeral variant tendon extends to join the flexor carpi ulnaris tendon shortly before its insertion (long arrow). Palmaris longus is present in the right forearm (p). B: Closer view of humeral variant (large arrowhead) and flexor carpi ulnaris (small arrowhead) tendons and insertion shown in A. An extra small muscular slip (asterisk) extends from the base of the hypothenar eminence. C: Closer view of the extratendinous slip (long

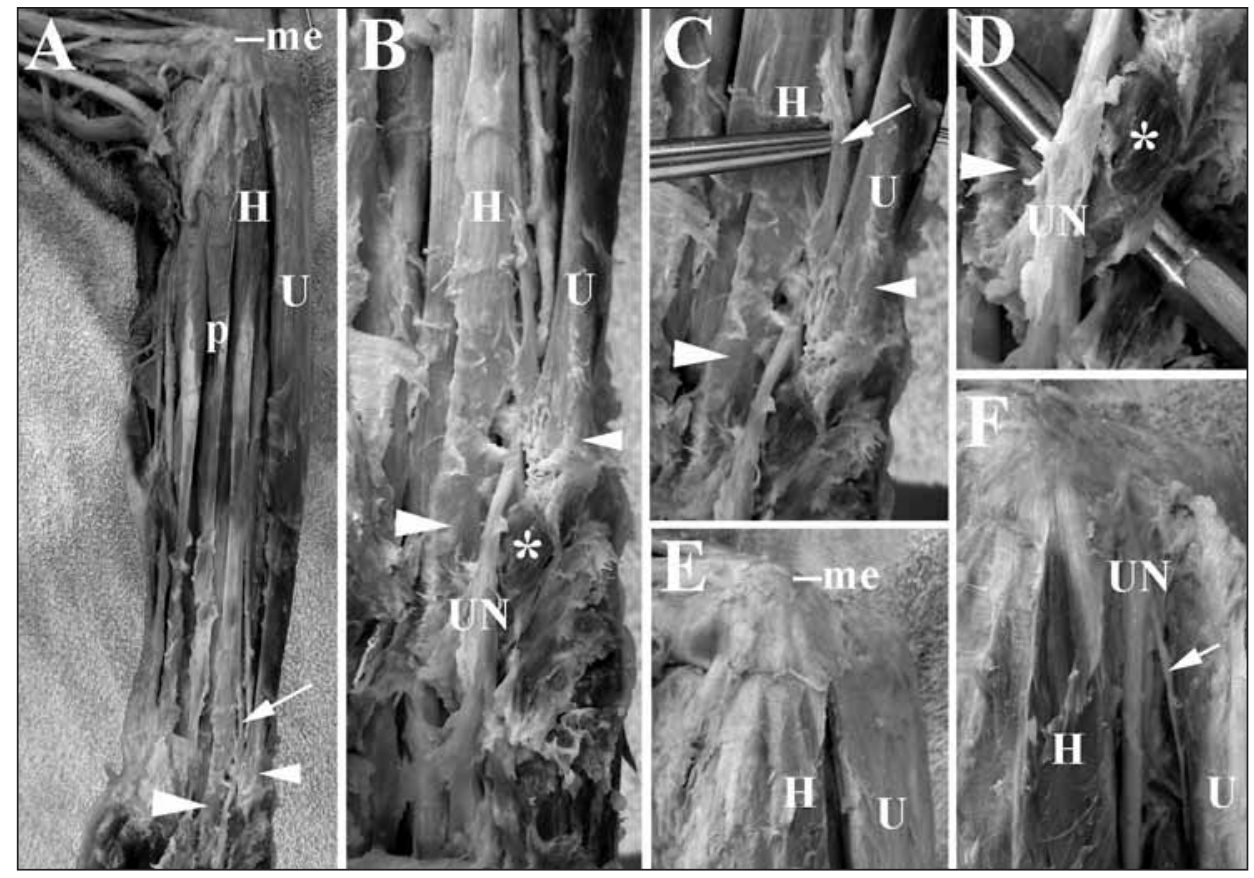

arrow) shown in A passing from the humeral variant (large arrowhead) to the flexor carpi ulnaris tendon (small arrowhead). D: Closer view of extra muscular slip (asterisk) shown in B that extends toward insertion of the humeral variant tendon (large arrowhead). E: Closer view of variant right flexor carpi ulnaris origin. F: Reflection of the ulnar head from the medial epicondyle shows ulnar nerve passing between humeral variant and flexor carpi ulnaris with a branch passing to the ulnar portion (short arrow). H: humeral variant; U: ulnar belly of flexor carpi ulnaris; me: medial epicondyle; UN: ulnar nerve; UA: ulnar artery. 
ulnaris is similar to that reported by Rao et al. ${ }^{3}$ with the exception noted here that tendons of the humeral and ulnar heads remained separated until their insertion on the pisiform rather than fusing prior to insertion. In addition, there appeared a tendinous slip that passed from the humeral tendon to insert separately on the hamate not observed in the previously reported variant. In the present case, origin of the flexor carpi ulnaris on the right side on the right side was unusual in that there was little if any representation of the humeral head and the muscle was essentially ulnar in origin. The anomalous humeral addition originating from the medial epicondyle had similar muscular characteristics to the humeral head of flexor carpi ulnaris on the left except that its innervation appeared pimarily from the median nerve and its insertion was on the hamate. Other right side variations were also noted including a small extratendinous band that extended from the humeral variant tendon to join the flexor carpi ulnaris tendon just before its insertion and the appearance of an extra muscular slip extending from the pisiform to the insertion of the variant humeral tendon on the hamate.

Previous reports of anomalous muscles in the medial aspect of the forearm have been described including a case originating from the distal flexor carpi ulnaris extending to flexor digiti minimi ${ }^{6}$ and also an extra accessory muscle in addition to the usual flexor carpi ulnaris with origin from the medial epicondyle and antebrachial fascia that passed to the pisiform. ${ }^{8}$ As noted in the first report of separate humeral and ulnar bellies of the flexor carpi ulnaris, ${ }^{3}$ origin of the two heads of flexor carpi ulnaris in the left forearm in the present instance was unlike those of other reported muscular anomalies in the anterior compartment, suggesting that the variant muscle bellies in this case represent medial and lateral compartments of flexor carpi ulnaris that did not fuse during early appendicular development and formed separate tendons. $^{3,9,10}$

In the right forearm it is possible that due to insertion on the hamate and innervation by the median nerve the humeral variant simply represented an aberrant muscle addition and thus was distinct from the flexor carpi ulnaris. On the other hand, because of only minor con- nection from the medial epicondyle to flexor carpi ulnaris and all musculature essentially associated with the ulnar belly, a muscular humeral head would have to be considered largely lacking. It is possible that due to similarity of its origin and muscle/tendon morphology to that of the humeral belly on the left, the extra muscle arising from the medial epicondyle might be considered to be a variant humeral head of flexor carpi ulnaris that also remained separate from the ulnar belly. Moreover, the extratendinous connection to that of flexor carpi ulnaris before insertion on the pisiform would also suggest relationship between the two muscles. In either case, although innervation and insertion of this numeral addition was different from the humeral belly of the normal flexor carpi ulnaris, basic function of wrist flexion and medial deviation would be preserved with this muscular variation.

An interesting and potentially clinically relevant variation associated with this case was the presence of extratendinous slips extending from the humeral belly of flexor carpi ulnaris to the hamate in the left forearm and from the humeral variant to the flexor carpi ulnaris tendon on the right. On the right side, the superficial branch of the ulnar nerve emerged just distal to the extratendinous band and between the insertion of the humeral variant and flexor carpi ulnaris tendons, in all likelihood not apt to contribute to ulnar neuropathy unless under extreme circumstances. In the left forearm, however, this tendinous band arched over and was in immediate proximity to the ulnar artery and superficial ulnar nerve. Compression or entrapment of vessel and/or nerve by the tendon variant reported here could be a potential cause for clinical problems in the palmar region. Indeed, previously reported tendon variants of flexor carpi ulnaris have caused ulnar nerve compression, including splitting of the ulnar nerve by lateral tendon duplication $^{5}$ and entrapment of the ulnar nerve by division of the insertion of the tendon on the pisiform ${ }^{7}$ resulting in significant ulnar neuropathies. Interestingly, earlier descriptions of flexor carpi ulnaris tendon variation also include extratendinous bands to the fourth and fifth metacarpals or carpal ligaments ${ }^{11}$ reminiscent of the variation noted here. 
Flexor carpi ulnaris and anomalous muscular/tendinous variations associated with the medial forearm have been reported previously, but in relatively few instances. The bilateral presence of muscular variations on the medial aspect of the anterior compartment of the forearm discovered here shortly after the recent report of a similar unilateral variant ${ }^{3}$ suggests that cases of anatomical variation of flexor carpi ulnaris may be more predominant than previously believed. As such, the documentation presented here underscores for teaching anatomists, clinicians and surgeons that they must be cognizant of these particular types of muscular variants and this knowledge will be of value to surgical strategies concerning the antebrachium and manus.

\section{References}

1. Woodburne RT. Burkel WE. Essentials of Human Anatomy. 8th ed. New York: Oxford University Press; 1988.

2. Georgiev GP, Jelev L, Ovtscharoff WA. Unusual combination of muscular and arterial variations in the upper extremity: a case report of a variant palmaris longus and an additional tendinous portion of the flexor carpi ulnaris together with a persistent median artery. Anatomy 2009; 3: 58-61.
3. Rao MRK, Bhat SM, Venkataramana V, Rodrigues V. Ulnar and humeral heads forming separate bellies. A rare case of digastric flexor carpi ulnaris muscle. Int 7 Morphol 2009; 27: 31-4.

4. Grechenig W, Clement H. Egner S, Tesch NP, Weiglein A, Peicha G. Musculo-tendinous junction of the flexor carpi ulnaris muscle. An anatomical study. Surg Radiol Anat 2000; 22: 255-60.

5. al-Quattan MM, Duerkson F. A variant of flexor carpi ulnaris causing ulnar nerve compression. F Anat 1992; 180: 189-90.

6. Pribyl CR, Moneim MS. Anomalous hand muscle found in the Guyon's canal at exploration for ulnar artery thrombosis. A case report. Clin Orthop Relat Res 1994; 306: 120-23.

7. Zook EG, Kucan JO, Guy RJ. Palmar wrist pain caused by ulnar nerve entrapment in the flexor carpi ulnaris tendon. 7 Hand Surg 1988; 13: 732-35.

8. Arnold G, Zech M. An accessory muscle and additional variants of the forearm. Handchirugie 1977; 9: 135-36.

9. Lim AY, Kumar VP, Hua J, Pereira BP, Pho RW. The neuromuscular compartments of the flexor carpi ulnaris. Plast Reconstr Surg 1999; 103: 1046-51.

10. Segal RL, Catlin PA, Krauss EW, Merick KA, Robilotto JB. Anatomical partitioning of three forearm muscles. Cells Tissues Organs 2002; 170: 183-97.

11. Testut L, Lataret A. Traite d'Anatomie Humaine, 8th ed. Paris: Gaston Doin; 1928.

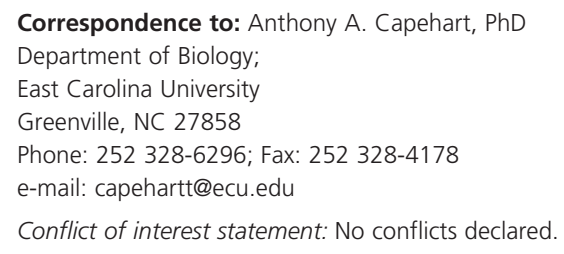

